\title{
Article \\ Influence of Magnetic Field on Amplification without Inversion Induced by a Femtosecond Pulse Train
}

\author{
Qianqian Ji ${ }^{1,2}$, Rui Ma ${ }^{1,2}$, Tianwei Jiang ${ }^{1,2}$ and Bin Luo ${ }^{1,2, *}$ \\ 1 School of Electronic Engineering, Beijing University of Posts and Telecommunications, Beijing 100876, China; \\ jiqian@bupt.edu.cn (Q.J.); marui_by@bupt.edu.cn (R.M.); jtw@bupt.edu.cn (T.J.) \\ 2 State Key Laboratory of Information Photonics and Optical Communications, Beijing University of Posts and \\ Telecommunications, Beijing 100876, China \\ * Correspondence: luobin@bupt.edu.cn
}

Citation: Ji, Q.; Ma, R.; Jiang, T.; Luo, B. Influence of Magnetic Field on Amplification without Inversion Induced by a Femtosecond Pulse Train. Appl. Sci. 2022, 12, 1780. https://doi.org/10.3390/ app12041780

Academic Editors: Guillaume Duchateau and Marco Anni

Received: 13 December 2021

Accepted: 7 February 2022

Published: 9 February 2022

Publisher's Note: MDPI stays neutral with regard to jurisdictional claims in published maps and institutional affiliations.

Copyright: (C) 2022 by the authors. Licensee MDPI, Basel, Switzerland. This article is an open access article distributed under the terms and conditions of the Creative Commons Attribution (CC BY) license (https:// creativecommons.org/licenses/by/ $4.0 /)$.

\begin{abstract}
Taking advantage of the short pulse duration and the high repetition rate of the fs pulse train, amplification without inversion (AWI) can be achieved in two- or three-level atomic systems. A modulation method using a static magnetic field to control such AWI gain in an atomic system is proposed. The schematic model of the $5^{2} S_{1 / 2}-5^{2} P_{1 / 2}$ transition of ${ }^{87} \mathrm{Rb}$ is adopted, and the results show that the system gain can be significantly enhanced and modulated by the magnetic field, while the population inversion can be almost completely suppressed. Moreover, the pulse number, pulse repetition period, and pulse area can also be utilized to modulate the AWI gain.
\end{abstract}

Keywords: fs pulse train; Zeeman effect; amplification without inversion

\section{Introduction}

Amplification without inversion (AWI) is the optical amplification that can be achieved with a smaller population in the upper level than the lower one, which is considered to be counter-intuitive to the traditional laser theory [1,2]. Up to now, various schemes based on quantum interference effects have been adopted to realize AWI, such as incoherent pumping [3-7], transient field controlling [8-10], microwave driving fields [11], etc. Recently, AWI has also been realized in nitrogen molecules using short, high-intensity pulses [12,13].

With pulse width on the scale of femtoseconds and repetition rates up to gigahertz, the fs pulse train can be used to manipulate the atomic transition process within its natural coherent time, thus allowing investigation of the transient characteristics and atomic coherent control therein. Many interesting phenomena have been demonstrated, such as electromagnetically induced transparency (EIT) [14], coherent population trapping (CPT) [15], and coherent population accumulation [16,17].

In this paper, we propose an AWI system induced by the fs pulse train in the presence of a static magnetic field. The theoretical results show that Zeeman splitting introduces an additional asymmetry and provides additional controllable parameters, which allow the modulation of the gain factor of AWI. The influence of the pulse train parameters, such as pulse number, pulse repetition period, and pulse area, is also analyzed.

\section{Theoretical Model}

To simplify the analysis, a two-level system with $m_{F}= \pm 1$ is adopted, which can be easily found in atoms, such as in the $5^{2} S_{1 / 2}-5^{2} P_{1 / 2}$ transition of ${ }^{87} \mathrm{Rb}$, as shown in Figure 1 . Under a static external magnetic field, a two-level system splits into a three-level $\Lambda$ system because of the Zeeman effect, which is the simplest structure for our study. Assuming that the probe field is weak enough not to affect the preparation of the atomic state, we then consider this three-level $\Lambda$ system interacting with the fs pulse train. The pulse train is assumed to be linearly polarized, which can be decomposed into left-circularly and 
right-circularly polarized components coupled with the transitions from $|1\rangle$ to $|2\rangle$ and $|3\rangle$ to $|2\rangle$, respectively. The Hamiltonian of this system is

$$
H=H_{0}+H_{\text {int }}
$$

where $H_{0}$ is the intrinsic Hamiltonian of the atomic system and $H_{\text {int }}=-\mu E(t)$, representing the interacting Hamiltonian where $\mu$ is the electric dipole moment and $E(t)$ is the electric field. Here, $E(t)$ can be expressed as:

$$
E(t)=\left[\sum_{n=0}^{N} \mathcal{E}\left(t-n T_{R}\right) e^{i n \phi_{R}}\right] e^{i \omega_{L} t}
$$

where $N$ is the number of pulses, $T_{R}$ is the pulse repetition period, $\phi_{R}=0$ is the phase shift, and $\omega_{L}$ is the central laser frequency. We assume the envelope of the pulse train to be Gaussian, $\varepsilon(t)=\hat{\varepsilon} E_{0} e^{-\pi\left(\frac{t}{\sigma}\right)^{2}}$, where $\hat{\varepsilon}$ is the polarization vector, $E_{0}$ is the peak electric field intensity, $\sigma=200 \mathrm{fs}$ is the pulse duration, and the Fourier limit of the frequency bandwidth is $2.205 \mathrm{THz}$. The terms $|1\rangle$ and $|3\rangle$ are Zeeman sublevels, and the shifts caused by the magnetic field B are (with $m_{F}= \pm 1$ ) $\Delta_{B}= \pm \frac{\mu_{B} m_{F} g_{F} B}{\hbar}$, Bohr magneton $\mu_{B}=9.274 \times 10^{-24} \mathrm{~J} / \mathrm{T}$, Lande factor $g_{F}=-\frac{1}{2}, \hbar=1.054 \times 10^{-34} \mathrm{~J} \cdot \mathrm{s}$, so $\Delta_{B}=(2 \pi \cdot 0.7 \mathrm{MHz} / \mathrm{G}) \times B$.

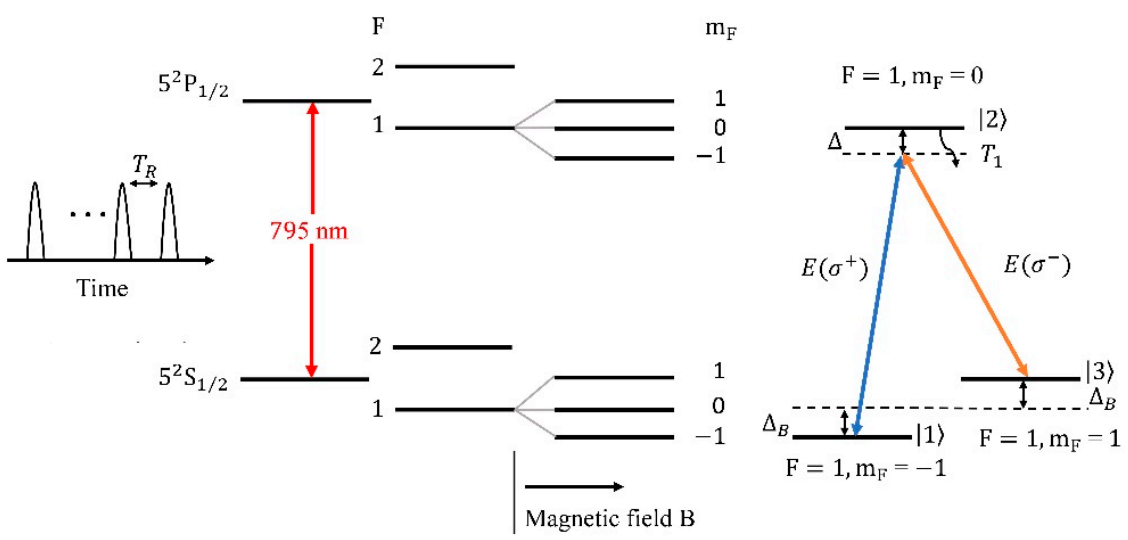

Figure 1. Theoretical scheme of two-level ${ }^{87} \mathrm{Rb}$ sublevels under Zeeman splitting. The nuclear spin quantum number of ${ }^{87} \mathrm{Rb}$ is $I=3 / 2$. The spontaneous relaxation time of ${ }^{87} \mathrm{Rb}$ is $T_{1}=28 \mathrm{~ns}$. The Zeeman splitting of the $\mathrm{F}=2$ sublevels is not shown. For $\mathrm{F}=1, g_{F}=-1 / 2$; when $\mathrm{B}=100 \mathrm{G}$, $\Delta_{B}=2 \pi \cdot 70 \mathrm{MHz}$

Under the rotating-wave approximation, the Hamiltonian of this system can be written as:

$$
H=\left(\begin{array}{ccc}
-\hbar\left(\Delta+\Delta_{B}\right) & -\frac{\hbar}{2} \Omega_{21}^{*} & 0 \\
-\frac{\hbar}{2} \Omega_{21} & 0 & -\frac{\hbar}{2} \Omega_{23} \\
0 & -\frac{\hbar}{2} \Omega_{23}^{*} & -\hbar\left(\Delta-\Delta_{B}\right)
\end{array}\right)
$$

where $\Delta$ is the detuning of the central laser frequency from the atomic transition frequency and $\Omega_{i j}=\frac{\mu_{i j} E_{0}}{\hbar}(i \neq j)$ is the Rabi frequency of the corresponding transition of levels $|i\rangle \leftrightarrow|j\rangle$. Assuming that the Rabi frequency is a real parameter, it can be considered that $\Omega_{21}=\Omega_{21}^{*}=\Omega_{23}=\Omega_{23}^{*}=\Omega$. The pulse area is expressed as $\theta=\int_{-\infty}^{+\infty} \Omega \frac{E(t)}{E_{0}} d t$. To describe the dynamic evolution of this system, we used the density matrix equation [18]:

$$
i \hbar \frac{\partial \rho}{\partial t}=[H, \rho]+\mathcal{L} \rho,
$$

where $\mathcal{L} \rho$ is the damping term in Langevin form. 
Combining Equations (3) and (4), the dynamical evolution of this system is given as:

$$
\begin{aligned}
& \frac{\partial \rho_{11}}{\partial t}=\frac{1}{T_{2}} \rho_{22}-\frac{i}{2} \Omega\left(\rho_{12}-\rho_{21}\right), \\
& \frac{\partial \rho_{12}}{\partial t}=-\frac{1}{T_{2}} \rho_{12}+i\left(\Delta+\Delta_{B}\right) \rho_{12}-\frac{i}{2} \Omega\left(\rho_{11}-\rho_{22}+\rho_{13}\right), \\
& \frac{\partial \rho_{13}}{\partial t}=2 i \Delta_{B} \rho_{13}-\frac{i}{2} \Omega\left(\rho_{12}-\rho_{23}\right), \\
& \frac{\partial \rho_{21}}{\partial t}=-\frac{1}{T_{2}} \rho_{21}-i\left(\Delta+\Delta_{B}\right) \rho_{21}+\frac{i}{2} \Omega\left(\rho_{11}-\rho_{22}+\rho_{31}\right), \\
& \frac{\partial \rho_{22}}{\partial t}=-\frac{1}{T_{1}} \rho_{22}+\frac{i}{2} \Omega\left(\rho_{12}+\rho_{32}-\rho_{21}-\rho_{23}\right), \\
& \frac{\partial \rho_{23}}{\partial t}=-\frac{1}{T_{2}} \rho_{23}-i\left(\Delta-\Delta_{B}\right) \rho_{23}+\frac{i}{2} \Omega\left(\rho_{13}-\rho_{22}+\rho_{33}\right), \\
& \frac{\partial \rho_{31}}{\partial t}=-2 i \Delta_{B} \rho_{31}-\frac{i}{2} \Omega\left(\rho_{32}-\rho_{21}\right), \\
& \frac{\partial \rho_{32}}{\partial t}=-\frac{1}{T_{2}} \rho_{32}+i\left(\Delta-\Delta_{B}\right) \rho_{32}-\frac{i}{2} \Omega\left(\rho_{31}-\rho_{22}+\rho_{33}\right), \\
& \frac{\partial \rho_{33}}{\partial t}=\frac{1}{T_{2}} \rho_{22}-\frac{i}{2} \Omega\left(\rho_{32}-\rho_{23}\right)
\end{aligned}
$$

where $\rho_{11}+\rho_{22}+\rho_{33}=1$. The spontaneous relaxation time of ${ }^{87} \mathrm{Rb}$ is $T_{1}=28 \mathrm{~ns}$ and $T_{2}=2 T_{1}$ [19]. The real part of the off-diagonal elements $\left(\rho_{21}\right.$ and $\left.\rho_{23}\right)$ represents dispersion, and the imaginary part represents absorption. Therefore, the gain of the atomic system is related to the imaginary part of the off-diagonal element.

Since the duration of each pulse in the pulse train is much shorter than the pulse repetition period $T_{R}$ and the atomic spontaneous relaxation time $T_{1}$ of $28 \mathrm{~ns}$, a semianalytical method developed in [20-22] was adopted to solve the above density matrix equations. In each period of the pulse train, this process is divided into two parts. During the interaction time $0<\tau \leq \sigma$, the atomic relaxation behavior, which is much slower than the pulse duration $\sigma$, is ignored and only the pulse excitation is considered which can be treated as an undamped Rabi process. During $\sigma<\tau \leq T_{R}$, the atom only experiences spontaneous emission. For every input pulse, the obtained solution $\rho$ from the previous pulse is taken as the initial state of the atomic system for the next pulse interaction, and then the iteration is carried out repeatedly to obtain atomic states of this system after the pulse train interacting with the atoms. For problems about interactions between atoms and short pulse trains, it was proven that the result of this kind of semi-analytical method is consistent with the numerical fourth-order Runge-Kutta method [23].

\section{Results and Discussion}

The optical properties of the atomic system can be controlled by changing the magnetic field strength. As magnetic field B increases, the population is redistributed between Zeeman sublevels $|1\rangle$ and $|3\rangle$, resulting in destructive quantum interference between the ground and excited states [24]. The absorption and population variation of the system are shown in Figures 2 and 3, respectively. The repetition rate of the pulse train is assumed to be $0.36 \mathrm{GHz}$, corresponding to $T_{R}=2.8 \mathrm{~ns}$. The repetition rate of the absorption with detuning is shown in Figure 2 and is dependent on the pulse repetition rate. Without the magnetic field, the AWI phenomenon also occurs when the fs pulse train interacts with the atomic system, but the system has a smaller gain. However, with the presence of the magnetic field, it is clearly shown that the AWI effect can be significantly enhanced by the magnetic field. Because of the assistance of the magnetic field, a larger population is transferred from level $|3\rangle$ to level $|1\rangle$ during the detuning where the gain $\left(G_{21} \propto \operatorname{Im} \rho_{21}\right)$ is maximized, and thus the population inversion $\left(\rho_{22}-\rho_{11}\right)$ is reduced, but the gain improves, as shown in Figure 3. Since $\operatorname{Im} \rho_{21}$ in Figure $2 \mathrm{a}$ and $\operatorname{Im} \rho_{23}$ in Figure $2 \mathrm{~b}$ are mirror-symmetric, the same conclusion for levels $|1\rangle$ and $|2\rangle$ are also available for levels $|3\rangle$ and $|2\rangle$. In order to describe the influence caused by the magnetic field more clearly, the variation of amplification and population inversion $\left(\rho_{22}-\rho_{11}\right)$ for different magnetic field strengths and laser detuning is illustrated in Figure 4. The magnetic field and detuning jointly affect the atomic system gain. When the magnetic field changes, the detuning at which the gain achieves its maximum also varies. As the maximum gain at different magnetic fields was not obtained at the same 
detuning value, a projection was used to verify the effects induced by the magnetic field (see Figure $4 b$,d). It can be concluded that as the magnetic field increases, the system gain improves while the population inversion decreases during the detuning where the gain is maximized.
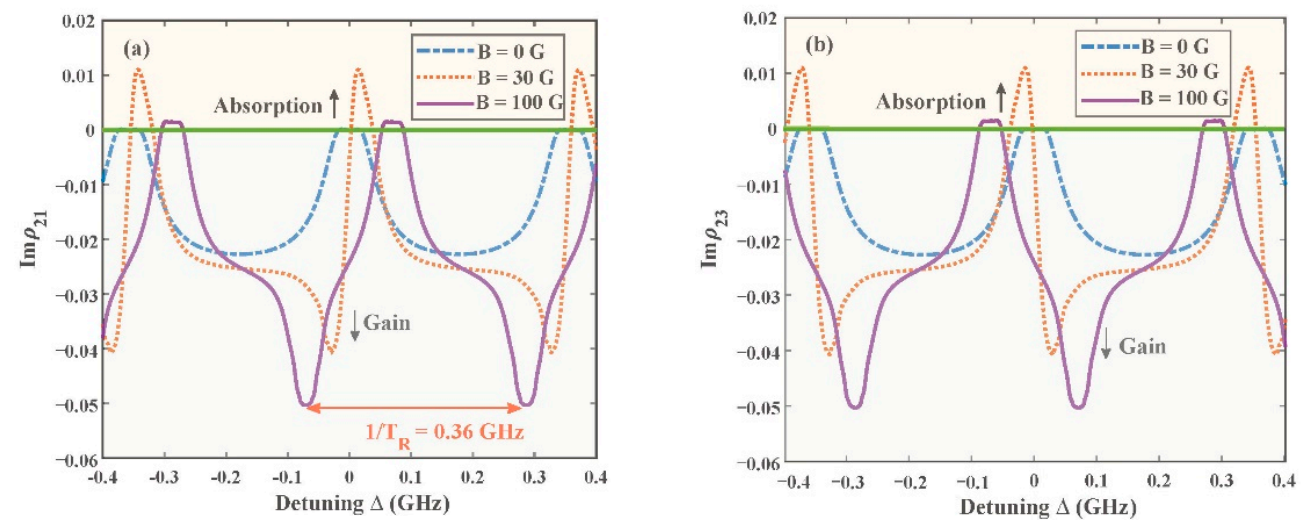

Figure 2. Variation of (a) $\operatorname{Im} \rho_{21}$ and (b) $\operatorname{Im} \rho_{23}$ with detuning for different magnetic fields. Other parameters are chosen as follows: $\mathrm{N}=400, \mathrm{~T}_{R}=2.8 \mathrm{~ns}$ and $\theta=\pi / 15$.
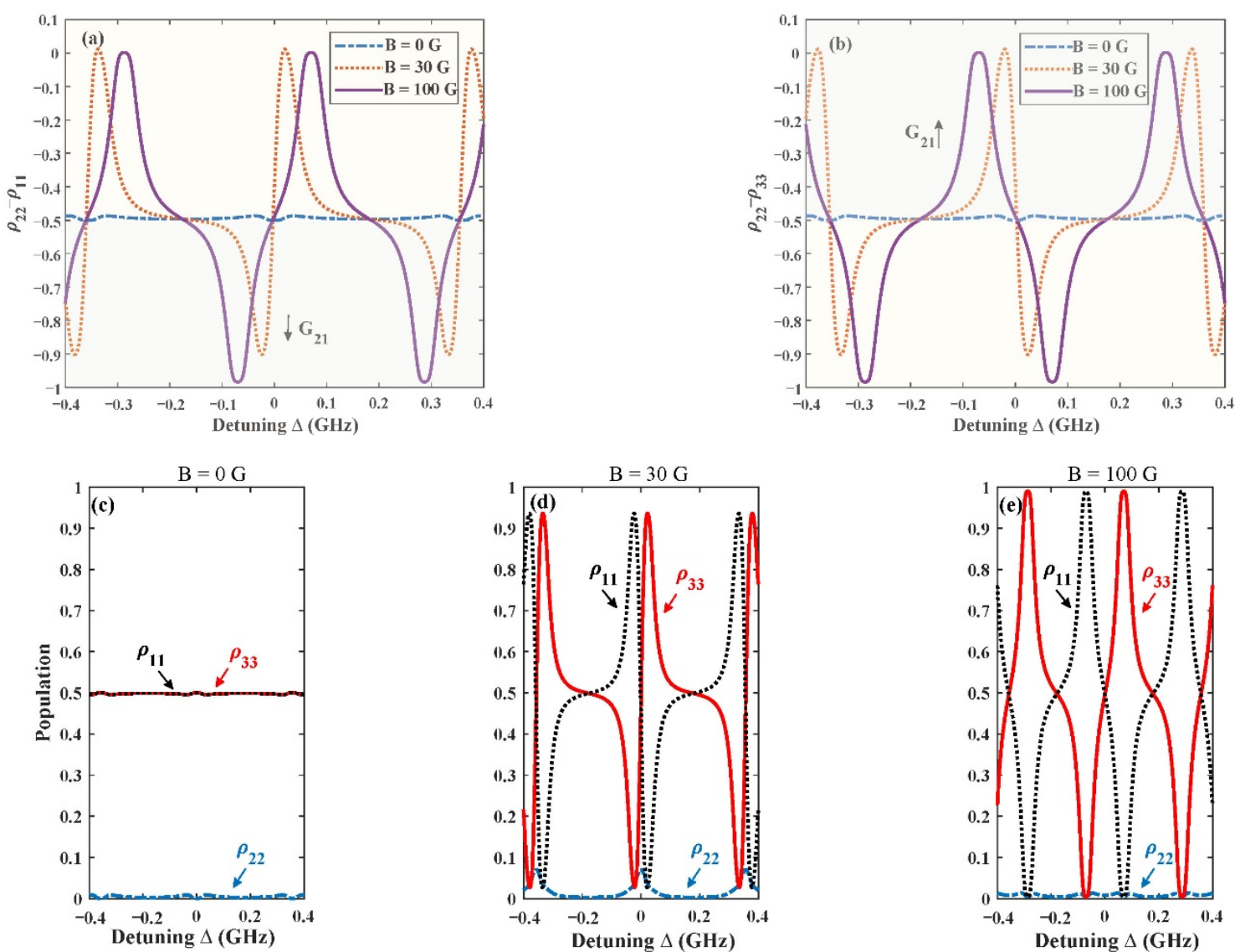

Figure 3. Variation of the population inversion of (a) $\left(\rho_{22}-\rho_{11}\right)$ and (b) $\left(\rho_{22}-\rho_{33}\right)$ with detuning at different magnetic fields. Variation of the population $\left(\rho_{22}, \rho_{33}, \rho_{11}\right)$ with detuning when $(\mathbf{c}-\mathbf{e})$ are $\mathrm{B}=0 \mathrm{G}, 30 \mathrm{G}, 100 \mathrm{G}$, respectively. Other parameters are the same as those in Figure 2.

The number of pulses is also an important parameter. The influence of whether the magnetic field is added to the system on the gain variation with the pulse number is shown in Figure 5. When there is no magnetic field, the system's gain gradually decreases as 
the pulse number increases. It should be noted that the system at a steady state has no gain, and all populations are equally distributed on ground state levels $|1\rangle$ and $|3\rangle$. In this case, the system no longer absorbs and amplifies the probe field, which can be considered the EIT phenomenon. However, with the presence of a magnetic field, the gain of the three-level system increases rapidly as the pulses accumulate, and it reaches its maximum after several cycles, while the population of the system remains almost completely in its ground state $|1\rangle$.
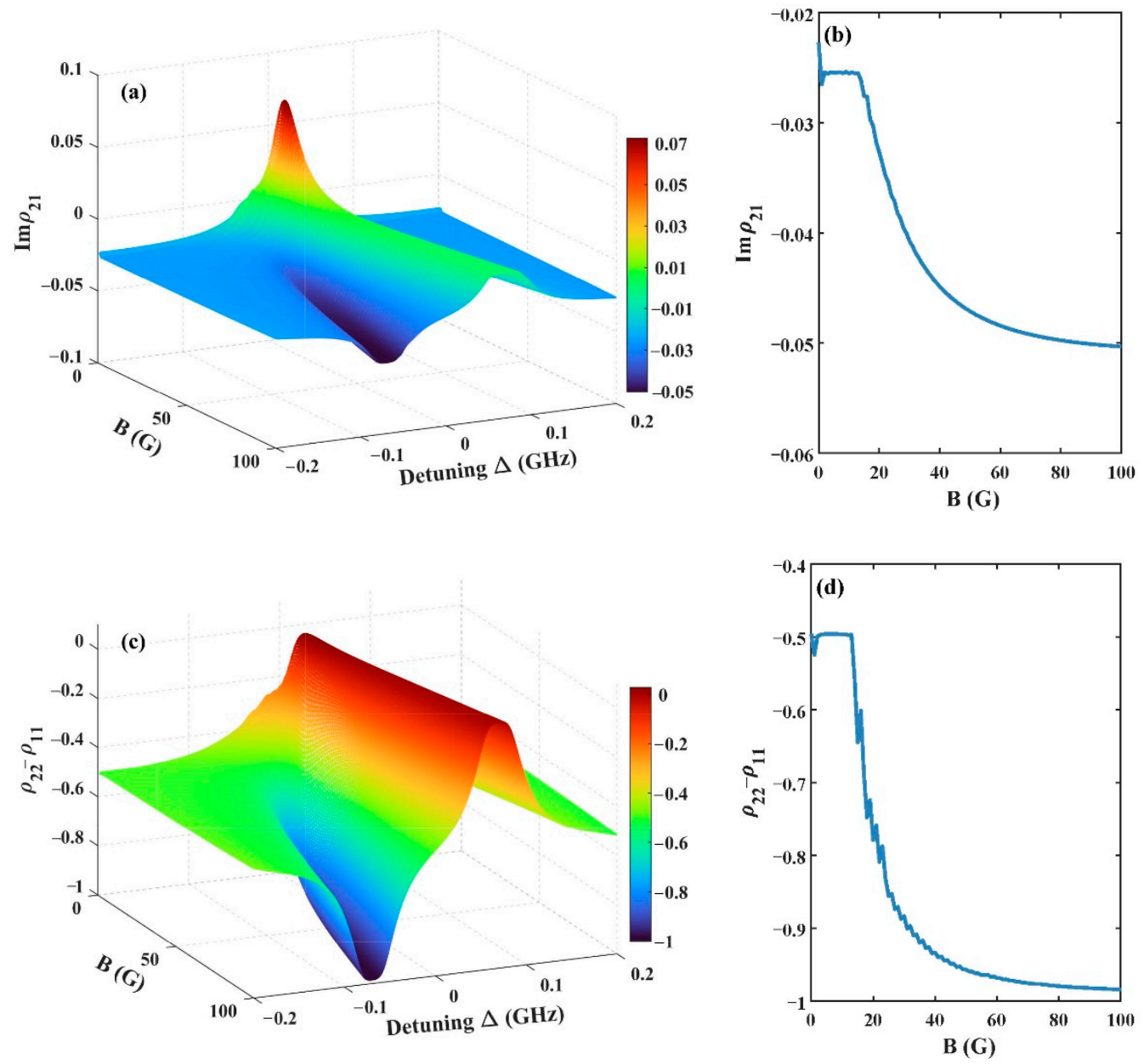

Figure 4. (a) Variation of the gain $\operatorname{Im} \rho_{21}$ with detuning and (b) the projection of the maximum gain $\operatorname{Im} \rho_{21}$ for different magnetic fields. (c) Variation of population inversion $\left(\rho_{22}-\rho_{11}\right)$ with detuning, and (d) the corresponding variation of population inversion at the maximum gain $\operatorname{Im} \rho_{21}$ for different magnetic fields. Other parameters are the same as those in Figure 2.

Then, we consider the influence of the pulse repetition period. As the pulse repetition period is reduced, the population inversion decreases, while the gain of the system improves. The magnetic field's influence on the variation of the amplification and population inversion of the system for different pulse repetition periods is shown in Figure 6. For a fixed relaxation time $T_{1}$, the smaller the repetition period, the greater population that can be excited within the relaxation time after a high enough number of pulses [23], which means a larger gain. Therefore, it can be concluded that the pulse repetition period is inversely proportional to the gain. Furthermore, the enhancement of AWI induced by the magnetic field remains obvious under different repetition periods. 

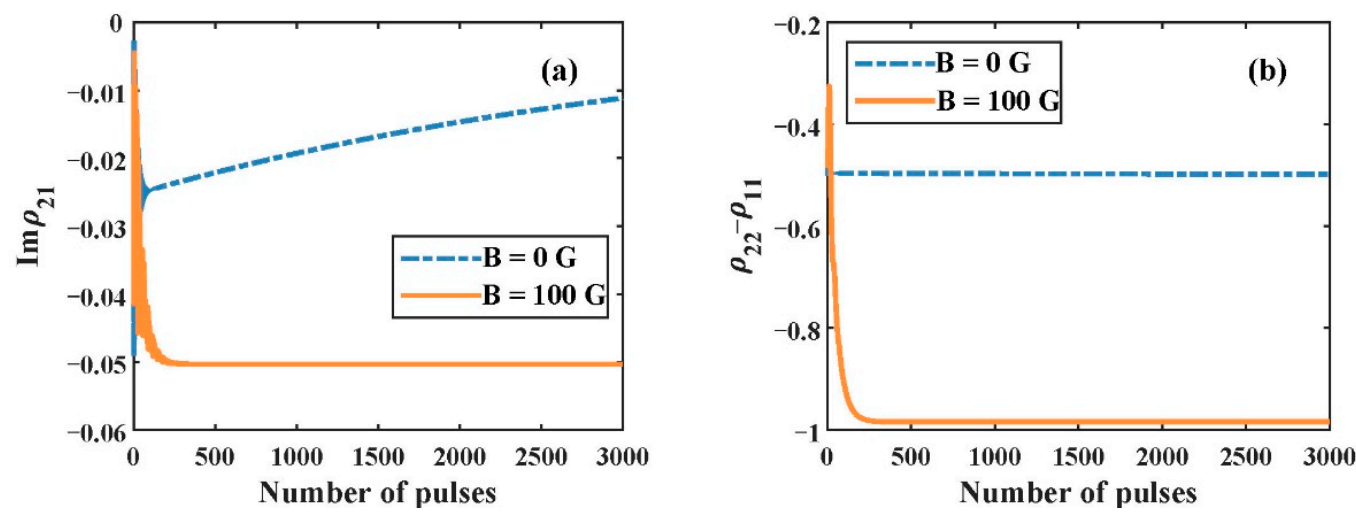

Figure 5. Evolution of (a) the maximum gain $\operatorname{Im} \rho_{21}$ and (b) the corresponding population inversion for levels $|1\rangle$ and $|2\rangle$ with the number of pulses for $B=0 \mathrm{G}$ and $\mathrm{B}=100 \mathrm{G}$. The other parameters are $\theta=\pi / 15$ and $T_{R}=2.8 \mathrm{~ns}$
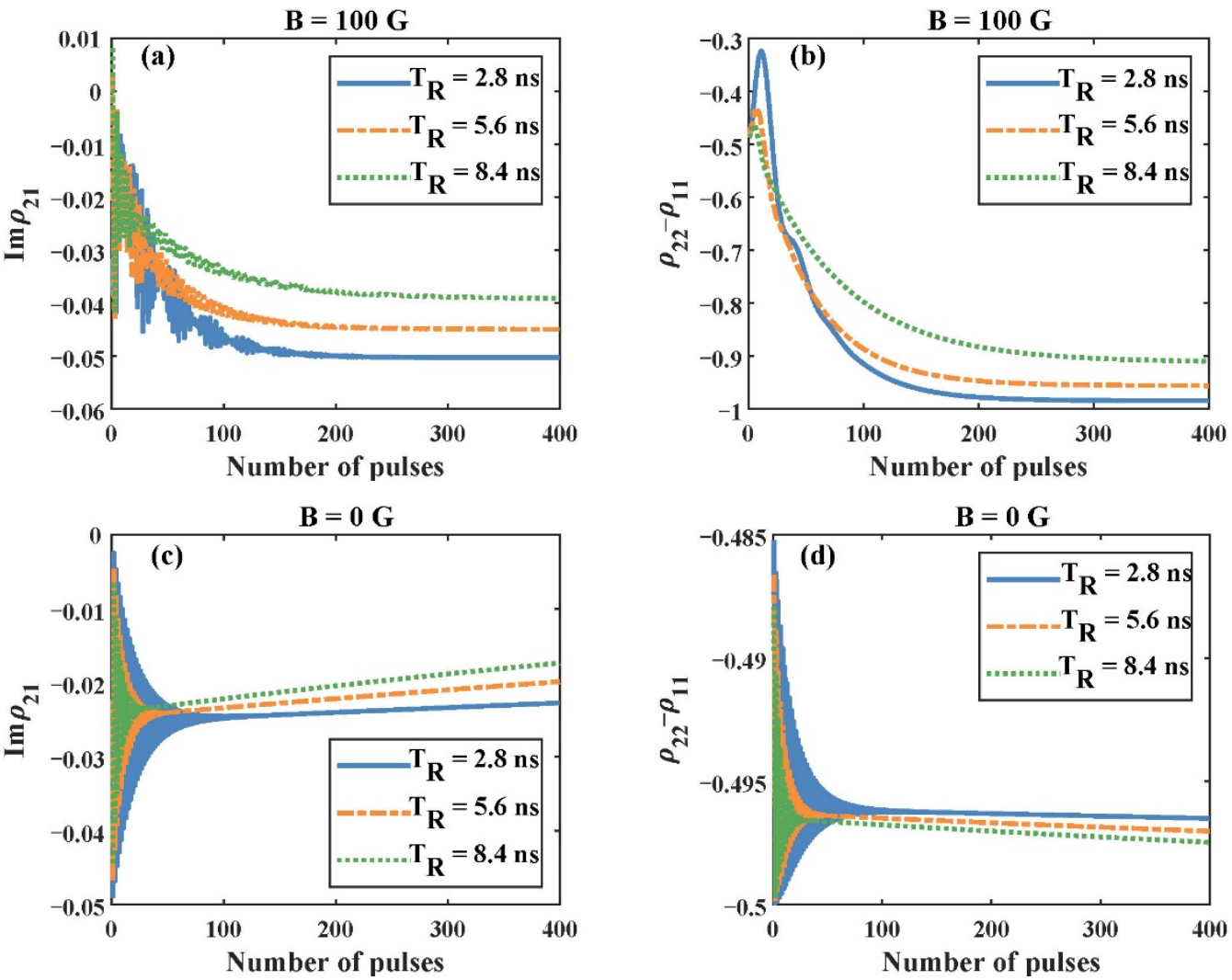

Figure 6. Evolution of the maximum gain $\operatorname{Im} \rho_{21}$ and the corresponding population inversion with the number of pulses for levels $|1\rangle$ and $|2\rangle$ at different pulse repetition periods ( $T_{R}=2.8 \mathrm{~ns}, 5.6 \mathrm{~ns}, 8.4 \mathrm{~ns}$ ) when $(\mathbf{a}, \mathbf{b}) \mathrm{B}=100 \mathrm{G}$ and $(\mathbf{c}, \mathbf{d}) \mathrm{B}=0 \mathrm{G}$. In above figures, $\theta=\pi / 15$.

Not only does the AWI change with different pulse repetition periods, the gain of the system can be also controlled by the pulse area of the fs pulse train. Without loss of generality, three different pulse areas are studied in detail, as shown in Figure 7. When the magnetic field is added, a conclusion similar to the previous discussion can be obtained, namely that the enhancement of AWI is still observed in different pulse areas. 

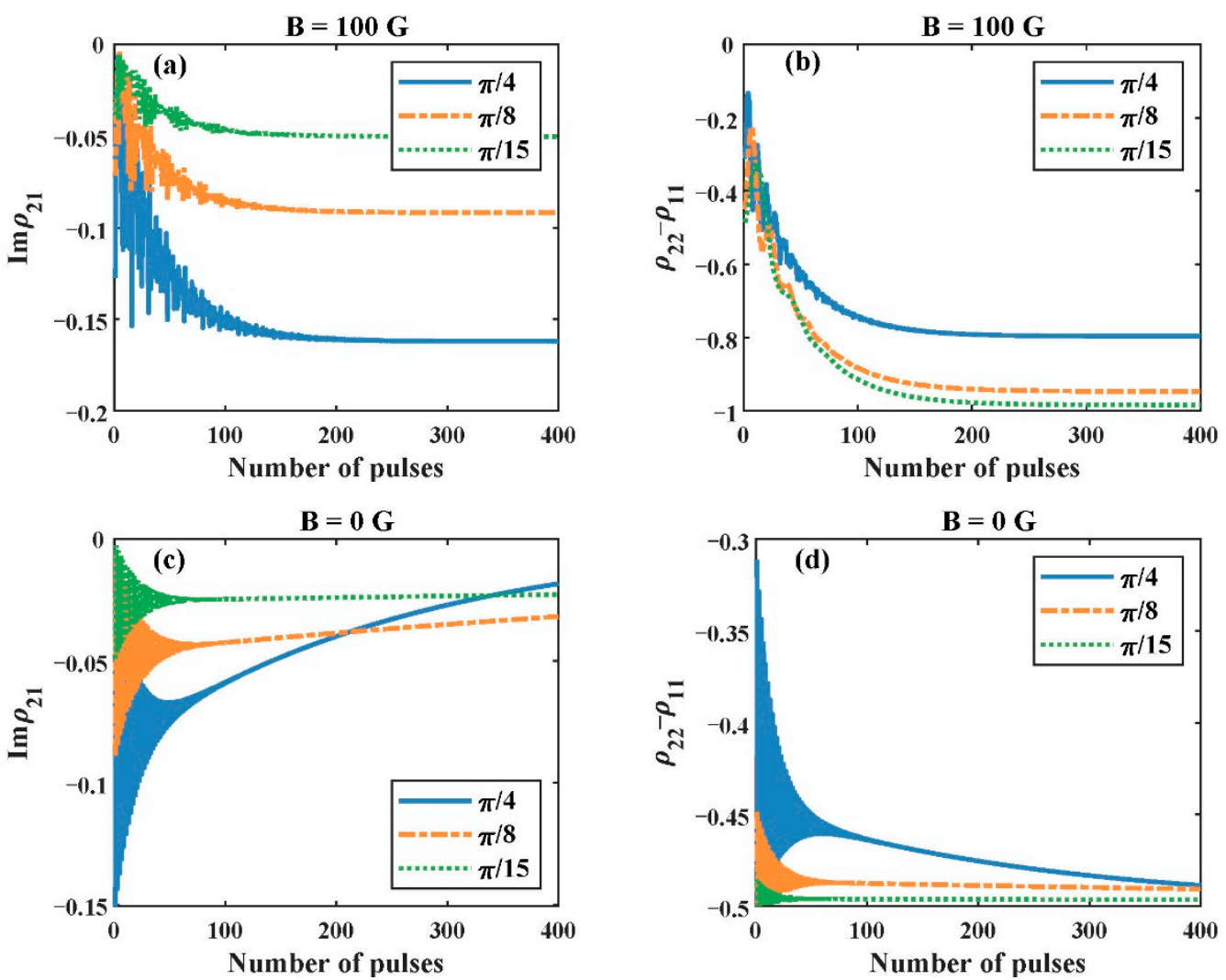

Figure 7. Evolution of the maximum gain $\operatorname{Im} \rho_{21}$ and the corresponding population inversion with the number of pulses for levels $|1\rangle$ and $|2\rangle$ at different pulse areas $(\theta=\pi / 4, \pi / 8, \pi / 15)$ when $(\mathbf{a}, \mathbf{b}) \mathrm{B}=100 \mathrm{G}$ and $(\mathbf{c}, \mathbf{d}) \mathrm{B}=0 \mathrm{G}$. In the above figures, $T_{R}=2.8 \mathrm{~ns}$.

\section{Conclusions}

In conclusion, the ${ }^{87} \mathrm{Rb}$ theory model was adopted, without loss generality, to investigate the AWI system induced by the fs pulse train with the presence of the magnetic field. By comparing the variation of AWI with and without the magnetic field, the theoretical analysis indicates that the gain of the system can be enhanced and modulated because of the contribution of the magnetic field. Furthermore, the effects of the pulse number, pulse repetition period, and pulse area on the AWI were also studied. The results show that the enhancement of AWI can be controlled by these parameters, while the enhancement due to the magnetic field remains significant. Therefore, in the atomic system, one can use not only the parameters of the fs pulse train to manipulate quantum states to perform modulation of the gain but also the assistance of the magnetic field. This result indicates an additional parameter to modulate such an AWI system.

Author Contributions: Conceptualization, Q.J. and B.L.; Data curation, Q.J. and R.M.; Formal analysis, Q.J., R.M. and B.L.; Funding acquisition, B.L.; Investigation: Q.J. and R.M.; Methodology, Q.J., R.M. and B.L.; Resources, T.J. and B.L.; Software: Q.J. and R.M.; Supervision: T.J. and B.L.; Validation: Q.J., T.J. and B.L.; Visualization, Q.J. and R.M.; Writing—original draft, Q.J.; Writingreview and editing, T.J. and B.L. All authors have read and agreed to the published version of the manuscript.

Funding: This work was supported by the National Natural Science Foundation of China (NSFC) (61771067).

Institutional Review Board Statement: Not applicable.

Informed Consent Statement: Not applicable.

Data Availability Statement: Not applicable. 
Conflicts of Interest: The authors declare no conflict of interest.

\section{References}

1. Kocharovskaya, O. Amplification and lasing without inversion. Phys. Rep. 1992, 219, 175-190. [CrossRef]

2. Mandel, P. Lasing without inversion: A useful concept? Contemp. Phys. 1993, 34, 235-246. [CrossRef]

3. Zibrov, A.S.; Lukin, M.D.; Nikonov, D.E.; Hollberg, L.; Scully, M.O.; Velichansky, V.L.; Robinson, H.G. Experimental demonstration of laser oscillation without population inversion via quantum interference in Rb. Phys. Rev. Lett. 1995, 75, 1499-1502. [CrossRef]

4. $\quad \mathrm{Wu}$, J.H.; Gao, J.Y. Phase control of light amplification without inversion in a $\Lambda$ system with spontaneously generated coherence. Phys. Rev. A 2002, 65, 063807. [CrossRef]

5. Jha, P.K. Effective unidirectional pumping for steady-state amplification without inversion. J. Mod. Opt. 2011, 58, 1957-1965. [CrossRef]

6. Sun, Z.; Jia, F.D.; Lv, S.F.; Ruan, Y.P.; Qing, B.; Xue, P.; Xu, X.Y.; Dai, X.C.; Zhong, Z.P. The Inversionless Amplification in a Tripod System of ${ }^{87} \mathrm{Rb}$ Atoms in a Magneto-optical Trap. Chin. Phys. Lett. 2014, 31, 043201. [CrossRef]

7. Al-Nashy, B.; Abdullah, M.; Al-Shatravi, A.G.; Al-Khursan, A.H. Lasing without population inversion in a four-level Y-type configuration in double quantum dot system. Pramana 2018, 91, 74. [CrossRef]

8. Nottelmann, A.; Peters, C.; Lange, W. Inversionless amplification of picosecond pulses due to Zeeman coherence. Phys. Rev. Lett. 1993, 70, 1783-1786. [CrossRef]

9. $\quad$ van der Veer, W.E.; van Diest, R.J.J.; Dönszelmann, A.; van Linden van den Heuvell, H.B. Experimental demonstration of light amplification without population inversion. Phys. Rev. Lett. 1993, 70, 3243-3246. [CrossRef]

10. Zhang, G.; Hu, S.; Li, J.; Jin, Y.; Liu, Y.; Li, S. Transient amplification without population inversion in a two-field-driven ladder-type three-level system. Optik 2020, 200, 163418. [CrossRef]

11. Scully, M.O.; Zhu, S.Y.; Gavrielides, A. Degenerate quantum-beat laser: Lasing without inversion and inversion without lasing. Phys. Rev. Lett. 1989, 62, 2813. [CrossRef]

12. Mysyrowicz, A.; Danylo, R.; Houard, A.; Tikhonchuk, V.; Zhang, X.; Fan, Z.; Liang, Q.; Zhuang, S.; Yuan, L.; Liu, Y. Lasing without population inversion in $\mathrm{N}_{2}^{+}$. APL Photonics 2019, 4, 110807. [CrossRef]

13. Richter, M.; Lytova, M.; Morales, F.; Haessler, S.; Smirnova, O.; Spanner, M.; Ivanov, M. Rotational quantum beat lasing without inversion. Optica 2020, 7, 586-592. [CrossRef]

14. Moreno, M.P.; Vianna, S.S. Coherence induced by a train of ultrashort pulses in a $\Lambda$-type system. JOSA B 2011, 28, 1124-1129. [CrossRef]

15. Aumiler, D. Coherent population trapping in ${ }^{87} \mathrm{Rb}$ atoms induced by the optical frequency comb excitation. Phys. Rev. A 2010, 82, 055402. [CrossRef]

16. Felinto, D.; Bosco, C.; Acioli, L.; Vianna, S. Coherent accumulation in two-level atoms excited by a train of ultrashort pulses. Opt. Commun. 2003, 215, 69-73. [CrossRef]

17. Felinto, D.; Acioli, L.H.; Vianna, S.S. Accumulative effects in the coherence of three-level atoms excited by femtosecond-laser frequency combs. Phys. Rev. A 2004, 70, 043403. [CrossRef]

18. Walls, D.F.; Milburn, G.J. Quantum Optics; Springer Science \& Business Media: New York, NY, USA, 2007.

19. Steck, D.A. Rubidium 87 D Line Data. 2001. Available online: https:/ / steck.us/alkalidata/ (accessed on 10 November 2021).

20. Soares, A.A.; de Araujo, L.E.E. Influence of propagation on the coherent accumulation of excitation induced by an ultrashort pulse train. Phys. Rev. A 2009, 80, 013832. [CrossRef]

21. Vujičić, N.; Ban, T.; Kregar, G.; Aumiler, D.; Pichler, G. Velocity-selective double resonance in Doppler-broadened rubidium vapor. Phys. Rev. A 2013, 87, 013438. [CrossRef]

22. Vujičić, N.; Kregar, G.; Ban, T.; Aumiler, D.; Pichler, G. Frequency comb polarization spectroscopy of multilevel rubidium atoms. Eur. Phys. J. D 2014, 68, 9. [CrossRef]

23. Soares, A.; de Araujo, L.E. Coherent accumulation of excitation in the electromagnetically induced transparency of an ultrashort pulse train. Phys. Rev. A 2007, 76, 043818. [CrossRef]

24. Dong, H.M.; Nga, L.T.Y.; Bang, N.H. Optical switching and bistability in a degenerated two-level atomic medium under an external magnetic field. Appl. Opt. 2019, 58, 4192-4199. [CrossRef] [PubMed] 